\title{
Fiber-optic fringe projection with crosstalk reduction by adaptive pattern masking
}

\author{
Steffen Matthias ${ }^{\mathrm{a}}$, Markus Kästner ${ }^{\mathrm{a}}$, and Eduard Reithmeier ${ }^{\mathrm{a}}$ \\ ${ }^{a}$ Institute of Measurement and Automatic Control, Leibniz Universität Hannover, Nienburger \\ Str. 17, 30167 Hannover, Germany
}

\begin{abstract}
To enable in-process inspection of industrial manufacturing processes, measuring devices need to fulfill time and space constraints, while also being robust to environmental conditions, such as high temperatures and electromagnetic fields. A new fringe projection profilometry system is being developed, which is capable of performing the inspection of filigree tool geometries, e.g. gearing elements with tip radii of $0.2 \mathrm{~mm}$, inside forming machines of the sheet-bulk metal forming process. Compact gradient-index rod lenses with a diameter of $2 \mathrm{~mm}$ allow for a compact design of the sensor head, which is connected to a base unit via flexible highresolution image fibers with a diameter of $1.7 \mathrm{~mm}$. The base unit houses a flexible DMD based LED projector optimized for fiber coupling and a CMOS camera sensor. The system is capable of capturing up to 150 gray-scale patterns per second as well as high dynamic range images from multiple exposures. Owing to fiber crosstalk and light leakage in the image fiber, signal quality suffers especially when capturing 3-D data of technical surfaces with highly varying reflectance or surface angles. An algorithm is presented, which adaptively masks parts of the pattern to reduce these effects via multiple exposures. The masks for valid surface areas are automatically defined according to different parameters from an initial capture, such as intensity and surface gradient. In a second step, the masks are re-projected to projector coordinates using the mathematical model of the system. This approach is capable of reducing both inter-pixel crosstalk and inter-object reflections on concave objects while maintaining measurement durations of less than $5 \mathrm{~s}$.
\end{abstract}

Keywords: fringe projection, image fibers, high dynamic range, inverse fringe projection

\section{INTRODUCTION}

Sheet-bulk metal forming is a new process, which is capable of producing parts with complex geometries by using a combination of deep drawing and bulk metal forming. ${ }^{1}$ Examples are parts featuring local gearing or carrier elements for transmission systems in the automotive industry. ${ }^{2}$ To ensure the quality of tools and produced parts of the process and gather data on wear mechanisms, adapted measuring devices are being developed. ${ }^{3}$ Owing to the constrained space inside the forming machine, classical measuring devices are not applicable for the in-process inspection task. In medical applications, endoscopes are commonly used for examinations of cavities. Recently, research has been focused on extending capabilities of endoscopic devices from 2-D to 3-D imaging. ${ }^{4}$ The measuring device presented in this paper for industrial applications uses a combination of optical fiber bundles and GRIN-lenses with fringe projection. Fringe projection is a fast method for measuring object geometries via triangulation and structured light patterns. By using flexible optical fiber bundles with a length of $1 \mathrm{~m}$, camera and projector can be housed in an external base unit to achieve a very compact design of the sensor head can be achieved. Attached to an extended arm, the compact sensor head allows for measuring tools inside forming machines.

As a result from different technical coatings applied to the tool surfaces, surface reflectance may very greatly, resulting in a high dynamic range of the fringe patterns captured by the camera. In combination with inter-core crosstalk in the fiber bundles, the high dynamic range of the fringe patterns leads to undesired interference when evaluating the phase of the patterns prior to triangulation of the 3-D geometry data.

Further author information:

E-mail: steffen.matthias@imr.uni-hannover.de, Telephone: +49 5117624456

Emerging Digital Micromirror Device Based Systems and Applications IX, edited by Michael R. Douglass, Benjamin L. Lee, Proc. of SPIE Vol. 10117, 101170A · @ 2017 SPIE CCC code: $0277-786 \mathrm{X} / 17 / \$ 18 \cdot$ doi: $10.1117 / 12.2254826$ 


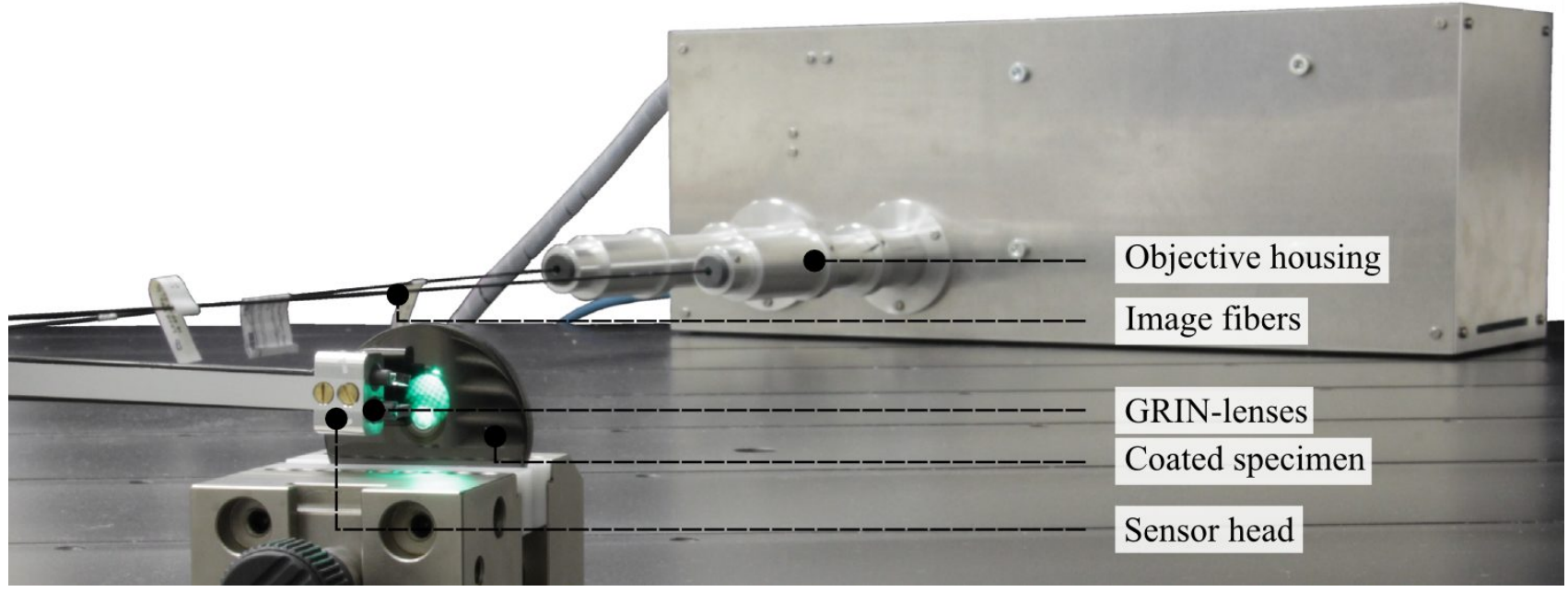

Figure 1. Fiber-optic fringe projection system.

After an introduction of the fiber-optic fringe projection system, the properties of the inter-core crosstalk of the employed fiber bundles are discussed. The measurement of the average point spread function enables a passive compensation of the fiber crosstalk by applying a deconvolution algorithm on the captured camera images. As an alternative, an active compensation method is presented, which masks out individual parts of the projector while capturing the HDR sequences in order to reduce the amount of crosstalk from overexposed areas in the individual captures. Results of measurements of a partially coated technical surface are shown, followed by a discussion of the advantages and disadvantages of both methods.

\section{FIBER-OPTIC FRINGE PROJECTION}

Figure 1 shows the fiber-optic fringe projection system with the sensor head in the foreground and the base unit in the background. The base unit contains both projector and camera of the fringe projection system. The system's projector is based on the DLP Discovery 4100 development kit in combination with a 0.7" DLP7000 micro-mirror matrix by Texas Instruments. The matrix features a resolution of $1024 x 768$ pixels and is capable of projecting 8 bit gray-scale patterns at a rate of $290 \mathrm{~Hz}$. Either a LED or a laser light source may be used for illuminating the projected patterns. ${ }^{5}$ A Point-Grey GS3-U3-23S6M-C industrial camera with a maximum frame-rate of $163 \mathrm{~Hz}$ is used to capture the projected patterns. Two optical fiber bundles type FIGH-100-1500N from Fujikura are used to couple the base unit to a compact sensor head. Both fibers feature 100.000 individual cores at a diameter of $1.7 \mathrm{~mm}$ and a length of $1.000 \mathrm{~mm}$.

Depending on the inspection task, different optics and lens arrangements may be used for the sensor head. The sensor head shown in Figure 1 measures $15 \times 10 \times 8.5 \mathrm{~mm}^{3}$ and features gradient refractive index (GRIN) lenses by GRINtech GmbH with a working distance of $10 \mathrm{~mm}$ combined with mirror prisms to change the angle of projection by 90 degrees. If a larger measuring volume is required, alternatively lenses with a working distance of $20 \mathrm{~mm}$ are available.

The system is capable of capturing images with a high dynamic range (HDR) by either combining an exposure series $^{6}$ of a projected pattern or controlling the current of the light source. Directly controlling the current of the light source can reduce the time required for capturing a HDR image. However, the intensity cannot be controlled linearly by current due to the use of a non-temperature stabilized LED and its nonlinear output.

In order to perform 3-D measurements, a sequence of sinusoidal patterns with defined phases and frequencies is projected onto the specimen. Following an initial calibration of the system, metric point cloud data can be calculated from the measured phase-maps in a triangulation step. 


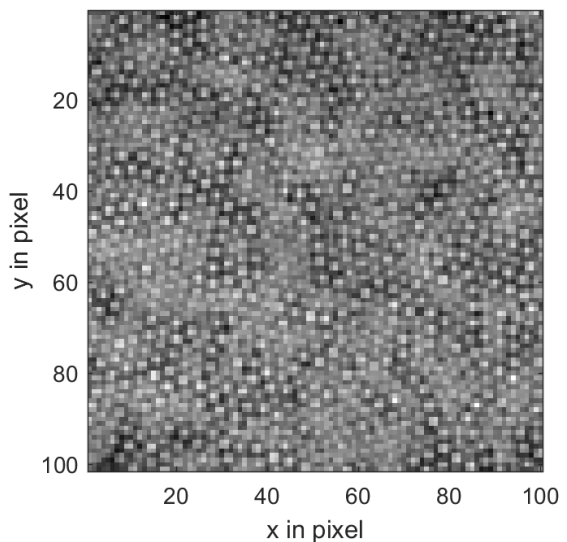

Figure 2. Close-up image of the fiber structure.

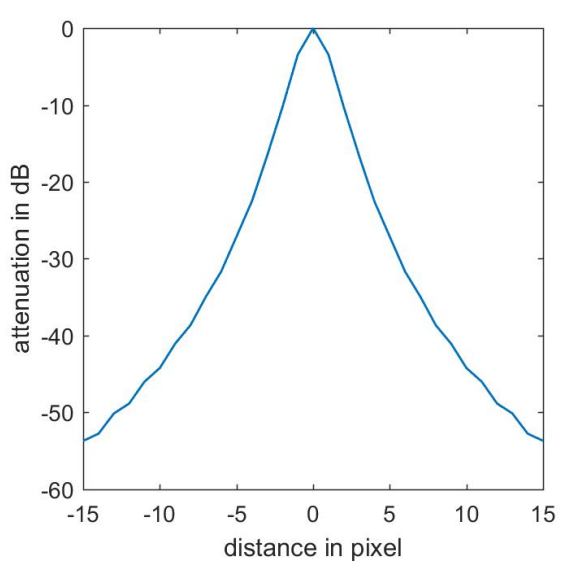

Figure 3. Cross section of the circular symmetric PSF.

\section{CROSSTALK IN IMAGING FIBER BUNDLES}

The fibers used for the fiber-optic fringe projection system consist of 100.000 individual cores, which are combined in a common cladding. The cores are ordered, meaning that the end faces are mirrored to each other. A reconstruction step to resolve the fiber order, which is necessary for incoherent imaging fibers, ${ }^{7}$ is therefore not required. Owing to the high density of fiber cores, there is however a significant amount of inter-fiber coupling or crosstalk, ${ }^{8}$ limiting the transmission properties of the fibers. ${ }^{9}$ Figure 2 shows a close-up image of the fiber bundle's grid structure. It can be seen that the fiber grid features a irregular structure. Due to the stochastic position of each core, the PSF for the described fiber bundles can be averaged to a circular symmetric PSF. The point spread function has been measured by directly coupling the projector unit to the camera unit with one of the fiber bundles. By projecting a one-dimensional frequency sweep sequence in both principal axes of the projector matrix, the magnitude response has been measured. ${ }^{10}$ The PSF is obtained from the magnitude response using the inverse Fourier transform and assuming zero phase.

Figure 3 shows a cross-section of the circular symmetric averaged 2-D point spread function (PSF). The average period of the fiber structure, which includes the cladding around the cores, is 2.88 pixels. On a local level this approximation is not accurate as each core only has a discrete number of neighboring cores and thus not a circular symmetric PSF. Additionally, the amount of crosstalk on a local scale is dependent on the varying distance and shape of the individual cores. On a global scale, the crosstalk exhibits a low frequency component with a wavelengths larger than the fiber bundle diameter, which is not accurately measured due to the low resolution of the DFT at these frequencies.

The reconstruction of the phase-map is in general robust to convolution with a symmetric PSF, for example from slight defocus, when measuring mostly diffuse reflective specimens. However, larger phase distortions occur when the amplitude of the projected patterns is modulated by the varying reflectance of a mostly specular reflective specimen.

\section{PASSIVE COMPENSATION}

As described in Section 3, crosstalk effects can be modeled via averaged point spread functions. This allows for compensation by the use of deconvolution algorithms on the captured images of a phase measuring sequence. Deconvolution is performed using the Richardson-Lucy (RL) algorithm ${ }^{11}$ for HDR sequences captured with the fiber-optic fringe projection system. In order to avoid amplifying noise present in the camera images, the number of iterations of the RL-algorithm is limited to 3. Alternatively regularized versions of the RL-algorithm may be used.

Experimental evaluations show that the used GRIN optics also have a significant influence on the measurement results. Deconvolution is thus performed using a combined PSF model of the fiber bundle and the GRIN optics. 


\section{ACTIVE COMPENSATION}

As an alternative to the reduction of crosstalk by modeling and deconvolution algorithms, and active method has been developed. The new algorithm aims to capture an accurate phase-map of the object by taking images at different exposures, similar to capturing an HDR image. Previous research demonstrates the effectiveness of HDR imaging for a two-camera fringe projection system. ${ }^{12}$ More research on the topic resulted in an iterative algorithm, which aims to reduce the dynamic range of the camera images by modulating the amplitude of the projected fringe patterns. ${ }^{13}$ The approach is however limited by the dynamic range of the structured light projector, which is often exceeded by pattern projected onto technical surfaces.

The adaptive algorithm evaluated in this manuscript is based on HDR imaging with a sequence of different exposure settings. In order to reduce crosstalk, a processing step is applied between the different exposures in the sequence. Based on an estimation of the camera pixel intensities in the next exposure iteration, saturated pixels on the camera matrix are mapped to the projector matrix and masked on the projection patterns. Additionally, instead of combining the unprocessed camera images of the exposure sequence to a HDR image, the phase evaluation algorithm is applied after each exposure. This avoids phase distortions from potential nonlinearities, for example from a current-controlled LED in the projector. The total number of pictures to be captured is identical to taken an HDR image. However, in between the individual images of the pattern sequence a processing step is required to adaptively calculate projector masks.

Input to the algorithm is a sequence of exposure configurations, e.g. the exposure durations of the camera or the LED current of the projector. Output is a camera phase map which describes projector and camera correspondences for subsequent triangulation. Each iteration consists of the following steps, with the exposure being increased from low to high:

1. Apply camera/projector exposure configuration for current iteration

2. Capture images of the masked phase-shift sequence

3. Calculate per-pixel phase values

4. Update phase-map with values of non-masked pixels

5. Estimate saturated camera pixels for next exposure step

6. Calculate projector and camera masks for next iteration

During the first step, either the camera exposure time or LED current of the projector is set to the next higher value in the sequence. For the first phase-sequence captured, no projector pixels are masked. Therefore, the configuration for the first exposure should be chosen to avoid any saturated camera pixels.

In the following step, the phase values for each camera pixel are calculated. ${ }^{14}$ The phase-map is updated with values which are not calculated from saturated pixels or pixels which are masked on the projector.

Saturated pixels for the exposure configuration in the next iteration are estimated by multiplying the sum of fringe amplitude and mean intensity in last captured images by the ratio of camera exposure times or LED current configurations.

Camera pixels which are estimated to be saturated in the next iteration are mapped to the projector. Depending on the specimen, this can be performed using the phase-map based on the epipolar constraint for stereo configurations. ${ }^{15}$ If the object is concave and the captured pattern images suffer from inter-object reflections, this approach might however lead to invalid mappings. In this case, a second pattern sequence rotated by 90 degrees needs to be projected to find the corresponding pixels on the projector matrix. The generated projector mask is additionally filtered using a 5x5 Gaussian kernel to avoid steep intensity gradients in the projected patterns, which potentially result in artifacts in situations where the specimen is slightly out of focus.

In general, the process is similar to capturing an HDR-image based on an exposure series. The additional processing time compared to a conventional HDR exposure series, resulting from the phase calculation and camera-projector mapping, amounts to less than $100 \mathrm{~ms}$ on an Intel-based desktop computer. 


\section{RESULTS}

To evaluate the effectiveness of both approaches in removing the crosstalk artifacts, measurements of both a planar and a spherical object are presented. The geometry of the objects enable a comparison against a virtual reference obtained by fitting either a plane or a sphere to the data. While the planar object leads to crosstalk effects with mainly lower frequency components for sensor pixels with relatively low signal amplitudes, the spherical object exposes high-frequency crosstalk components in proximity to the specular highlight.

The first reference object is a planar metal surface coated with CrAlCN coating, which is a common surface treatment for metal forming. In order to introduce variations in the surface reflectance, a circular spot on the surface has been left uncoated. While the coated surface area is mostly specular reflective, the uncoated spot is mostly diffuse reflective. This results in high intensity for the uncoated area, as seen in Figure 5, and low intensity for the coated areas in the captured fringe images.

The second reference object used in the evaluations is a tactile ball probe with a radius of $1 \mathrm{~mm}$, which has been treated by plasma ion nitriding. As a result from the convex surface, the intensity in the camera image has a single spike where the specular reflection hits the sensor, as shown in Figure 4.

Measurements have been performed for both objects using the uncompensated pattern projection approach, using deconvolution of the fiber and GRIN model (see Section 4), using adaptive pattern masking (see Section 5 ), as well as using a combination of deconvolution and adaptive masking. For the measurements of the planar specimen, all evaluations have been performed using $2.5 \mathrm{~ms}, 50 \mathrm{~ms}$, and $500 \mathrm{~ms}$ exposure time for the projection. For the spherical object, $3.5 \mathrm{~ms}, 20 \mathrm{~ms}$ and $60 \mathrm{~ms}$ exposure durations have been used. As no inter-object reflections are encountered on this specimen, pattern sequences with 3 frequencies and 4 phase-offsets are projected, resulting in a total of 36 projected patterns per measurement.

Table 1 shows the standard deviation of the point to reference distances for the coated planar object. $1 \%$ of the points with largest deviations have been removed as outliers from the evaluation. Additionally, the point to reference distances for the uncompensated measurement is shown in Figure 7 and the measurement with both deconvolution and masking applied in Figure 9.

Table 1. Standard deviation from fitted plane for planar object.

\begin{tabular}{|c|c|c|c|}
\hline no compensation & deconvolution & masking & deconvolution and masking \\
\hline $6.1 \mu \mathrm{m}$ & $5.2 \mu \mathrm{m}$ & $4.6 \mu \mathrm{m}$ & $3.9 \mu \mathrm{m}$ \\
\hline
\end{tabular}

Table 2 shows the standard deviation of the point to reference distances for the spherical object. Similar to the evaluations of the planar object, $1 \%$ of the points with largest deviations have been removed as outliers from the evaluation. The corresponding point to reference measurements are shown in Figure 6 and Figure 8.

Table 2. Standard deviation from fitted sphere for spherical object.

\begin{tabular}{|c|c|c|c|}
\hline no compensation & deconvolution & masking & deconvolution and masking \\
\hline $3.4 \mu \mathrm{m}$ & $2.1 \mu \mathrm{m}$ & $3.4 \mu \mathrm{m}$ & $2.1 \mu \mathrm{m}$ \\
\hline
\end{tabular}

Figure 7 shows the influence of low-frequency crosstalk on fringe projection measurements. Periodic deviations from the best-fit plane are visible, resulting in an increased standard deviation of the point to plane distances. Table 2 shows a significant reduction of the standard deviation for the measurements with deconvolution of the PSF applied. As discussed in Sections 3 and 4, this approach is however limited in its effectiveness in compensating the low-frequency crosstalk and to a lesser amount to local deviations from the globally averaged PSF. The active pattern masking approach is capable of reducing the artifacts even further. Best results are achieved by combining both methods.

In comparison, the results in Table 2 for the spherical object show an even larger relative improvement from the passive deconvolution approach. However, no improvement is visible from the adaptive masking, as a result from the very steep intensity gradient in the neighborhood of the highlight. 


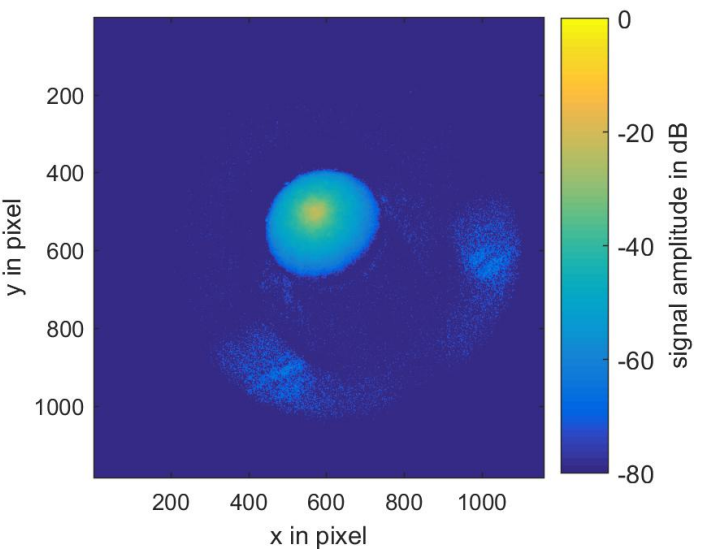

Figure 4. Signal intensity for spherical object.

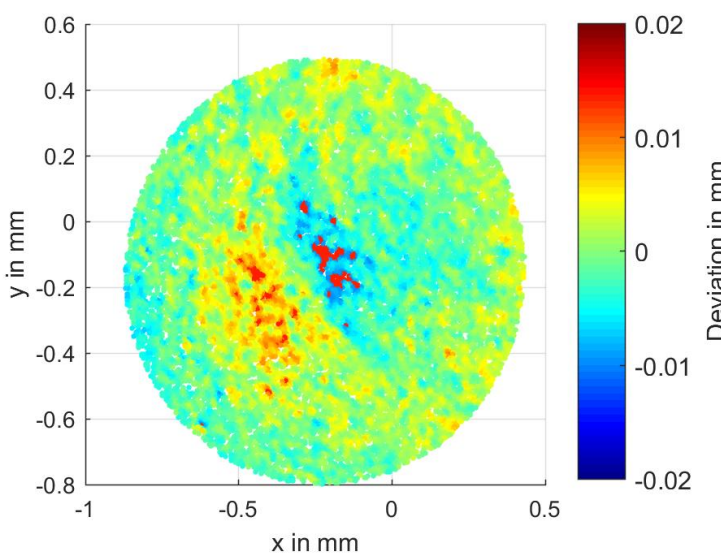

Figure 6. Deviations from sphere without compensation.

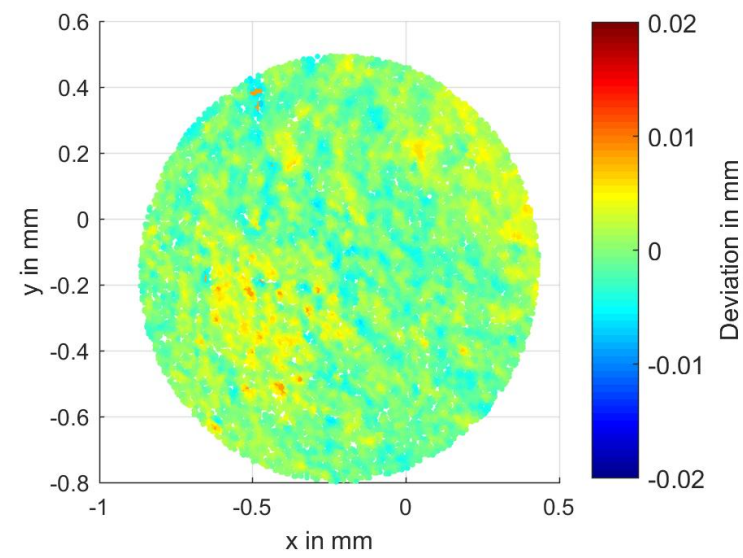

Figure 8. Deviations from sphere with compensation.

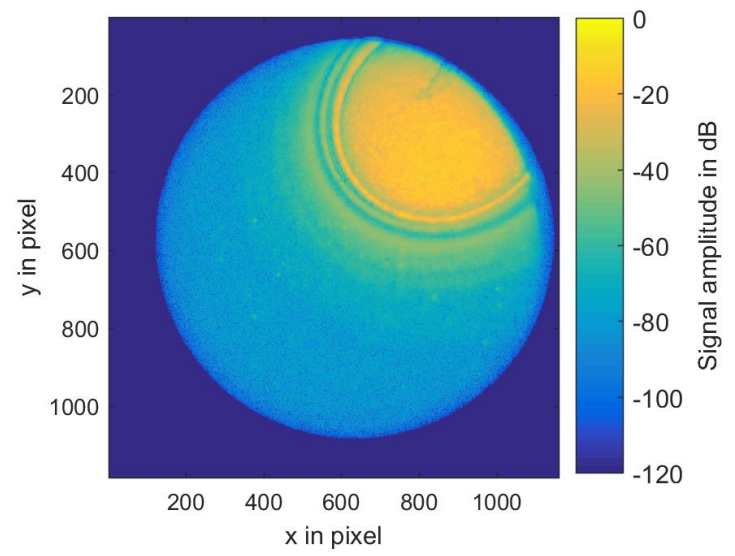

Figure 5. Signal intensity for partially coated planar object.

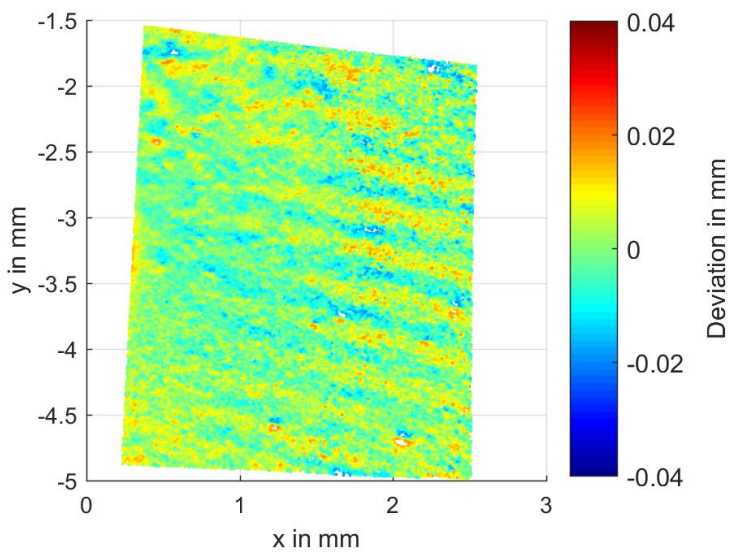

Figure 7. Deviations from plane without compensation.

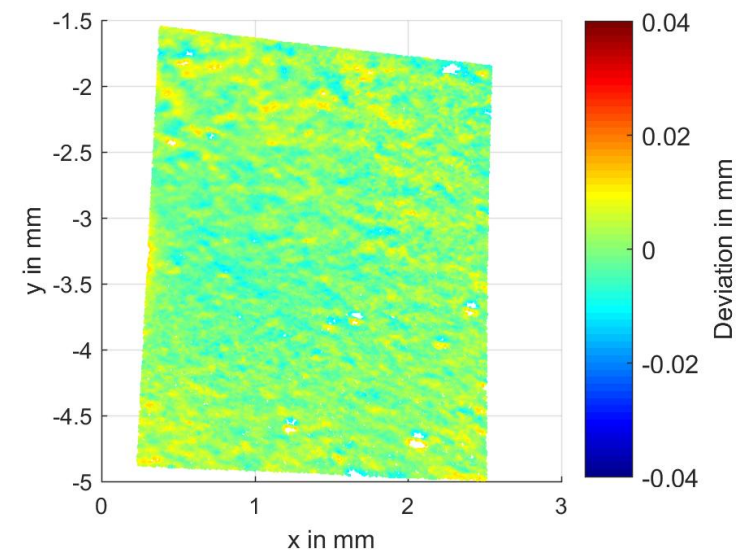

Figure 9. Deviations from plane with compensation. 


\section{DISCUSSION}

The results in Section 6 show a significant reduction in the point to reference standard deviation for both chosen specimens by using the presented compensation methods, resulting in a higher sensitivity of the device for the detection of local deformations on forming tools. While the passive approach to compensating crosstalk effects appears to be effective in situations with smaller local highlights, as encountered for the spherical object, it is less effective in the removal of lower-frequency crosstalk artifacts due to limitations in the model and deconvolution algorithms. In comparison, the active compensation based on adaptive pattern masking shows larger improvements for the planar object, but no improvement for the spherical object. This is a result of the coarseness of the masking for each exposure steps in combination with large signal intensity gradients. In conclusion, best results are achieved when combining both techniques.

Compared to a conventional HDR exposure sequence for capturing the pattern image, the computational overhead for the active compensation amounts to less than $100 \mathrm{~ms}$ per exposure step. Additionally, it is flexible as no apriori knowledge of the specimen or optical path is required. The passive approach is currently adding a computational overhead of several seconds to the measurements, as the deconvolution algorithm needs to be applied to each image in the pattern sequence. In the future, the use of a graphics processor to apply the deconvolution algorithm can reduce the processing times significantly. ${ }^{16}$

\section{SUMMARY AND OUTLOOK}

A new fringe projection system for in-process inspection tasks has been presented, which is capable of measuring complex geometries in constrained spaces. Based on the measured average point-spread-function of the imaging fiber bundles, a passive approach to reduce artifacts from core-to-core crosstalk is presented. As an alternative, an algorithm based on capturing exposure sequences with adaptively masked patterns is described. By combining both approaches, crosstalk artifacts in measurements of highly reflective objects can be reduced. The standard deviation of point to reference deviations is reduced by more than $36 \%$ in the demonstrated situations.

Further research aims to detect inter-object reflections and solve the resulting ambiguity in the reconstruction by adaptively masking the projector patterns to reduce interference.

\section{ACKNOWLEDGMENTS}

The authors would like to thank the German Research Foundation (DFG) for funding the project B6 "Endoscopic geometry inspection" within the Collaborative Research Center (CRC) / TR 73. Special thanks to Dominic Stangier from TU Dortmund for providing the coated specimens used for the measurements in this manuscript.

\section{REFERENCES}

[1] M. Merklein, J. M. Allwood, B.-A. Behrens, A. Brosius, H. Hagenah, K. Kuzman, K. Mori, A. E. Tekkaya, A. Weckenmann, "Bulk forming of sheet metal," Annals of the CIRP 61, 725-745 (2012).

[2] Gröbel, D., Schulte, R., Hildenbrand, P., Lechner, M., Engel, U., Sieczkarek, P., Wernicke, S., Gies, S., Tekkaya, A. E., Behrens, B. A., Hübner, S., Vucetic, M., Koch, S., and Merklein, M., "Manufacturing of functional elements by sheet-bulk metal forming processes," Production Engineering 10(1), 63-80 (2016).

[3] Matthias, S., Loderer, A., Koch, S., Gröne, M., Kästner, M., Hübner, S., Krimm, R., Reithmeier, E., Hausotte, T., and Behrens, B.-A., "Metrological solutions for an adapted inspection of parts and tools of a sheet-bulk metal forming process," Production Engineering 10(1), 51-61 (2016).

[4] Geng, J. and Xie, J., "Review of 3-d endoscopic surface imaging techniques," Sensors Journal, IEEE 14(4), 945-960 (2014).

[5] Matthias, S., Kästner, M., and Reithmeier, E., "Comparison of laser and led illumination for fiber-optic fringe projection," SPIE Photonics Europe, 989905 (2016).

[6] Mann, S. and Picard, R. W., "On being 'undigital' with digital cameras: Extending dynamic range by combining differently exposed pictures," Proceedings of ISET , 442-448 (1995).

[7] Gamo, J., Demuynck, O., Esteban, Ó., Lázaro, J. L., and Cubillo, A., "Calibration of incoherent opticalfiber-bundles for image transmission purposes," Proc. of IADAT (2005). 
[8] Reichenbach, K. L. and Xu, C., "Numerical analysis of light propagation in image fibers or coherent fiber bundles," Optics Express 15(5), 2151 (2007).

[9] Chen, X., Reichenbach, K. L., and Xu, C., "Experimental and theoretical analysis of core-to-core coupling on fiber bundle imaging," Optics Express 16(26), 21598 (2008).

[10] Matthias, S., Kästner, M., and Reithmeier, E., "Modeling of imaging fiber bundles and adapted signal processing for fringe projection," Advanced Optical Technologies 5(5-6), 439-449 (2016).

[11] Richardson, W. H., "Bayesian-based iterative method of image restoration," Journal of the Optical Society of America 62(1), 55 (1972).

[12] Liu, G.-h., Liu, X.-Y., and Feng, Q.-Y., "3d shape measurement of objects with high dynamic range of surface reflectivity," Appl. Opt. 50(23), 4557-4565 (2011).

[13] Koninckx, T. P., Peers, P., Dutre, P., and van Gool, L., "Scene-adapted structured light," IEEE Computer Society Conference on Computer Vision and Pattern Recognition (CVPR'05) 2 (2005).

[14] Stahl, H. P., "Review of phase-measuring interferometry," San Dieg - DL Tentative, 704 (1990).

[15] Hartley, R. and Zisserman, A., [Multiple view geometry in computer vision], Cambridge university press (2003).

[16] Bruce, M. A. and Butte, M. J., "Real-time gpu-based 3d deconvolution," Opt. Express 21(4), 4766-4773 (2013). 